\title{
High-frequency electromagnetic radiation of germanium crystals in magnetic fields
}

\author{
G.V. Milenin, V.V. Milenin, R.A. Redko \\ V. Lashkaryov Institute of Semiconductor Physics, \\ NAS of Ukraine, \\ 41, prospect Nauky, 03680 Kyiv, Ukraine; \\ e-mail: milenin.gv@gmail.com
}

\begin{abstract}
The cyclotron radiation of plasma of thermal carriers of germanium crystals, which is not in the state of thermodynamic equilibrium with semiconductor, has been experimentally confirmed.
\end{abstract}

Keywords: cyclotron frequency, cyclotron radiation, semiconductor crystal.

Manuscript received 16.01.17; revised version received 14.04.17; accepted for publication 14.06.17; published online 18.07.17.

\section{Introduction}

Mobile charge carriers in semiconductor crystals in the state of thermal motion form solid-state plasma. Plasma placed in a magnetic field radiates electromagnetic waves due to the rotation of charged carriers with a cyclotron frequency under the action of the Lorentz force [1-3]. The cyclotron radiation of semiconductor crystals is of interest for at least the following two reasons. First, smooth tuning the radiation frequency is carried out by changing the magnetic field induction. Second, since the effective mass of charge carriers in semiconductor crystals can be tenth and hundredth fractions of the free electron mass, the cyclotron radiation frequency at available magnetic fields can lie in the microwave and far infrared (terahertz) ranges. The latter region of the wavelengths of electromagnetic waves is of some practical interest. This paper is devoted to investigation of cyclotron radiation inherent to thermal plasma of semiconductors.

\section{Experimental}

In experiments on the study of cyclotron radiation from semiconductor crystals, there was used a permanent magnet with an induction in the center of the air gap $B=$ $0.45 \mathrm{~T}$ and a cylindrical ingot of $p$-Ge, its height was $4.6 \cdot 10^{-2} \mathrm{~m}$, the base diameter was $2.9 \cdot 10^{-2} \mathrm{~m}$ and specific resistivity $0.44 \mathrm{Ohm} \cdot \mathrm{m}$. This ingot was placed into the air gap of the magnet in such a way that the axis of the cylindrical ingot was perpendicular to the vector of magnetic induction. The ingot itself was inside the resonator, which was an aluminum tube.

Registration of cyclotron radiation was carried out by using its effect on water. For this, 20 to $40 \mu \mathrm{l}$ of water were poured into the cylindrical plastic capsule. A thermocouple was fixed in the near-surface layer of water. In turn, the capsule was placed in a foam plastic cylinder that was inserted into the opposite end of the resonator. We used Keithley Model 2000 multimeter with $0.002 \%$ basic $\mathrm{DC}$ voltage accuracy for temperature measurements.

\section{Results and discussion}

In studies on the impact of the effect of cyclotron radiation from Ge light holes on water, both its heating and cooling were observed. It should be noted that the effect of liquid heating was not always clearly expressed.

So, Fig. 1 shows the results of heating a liquid with the volume $40 \mu \mathrm{l}$ by cyclotron radiation of a germanium ingot (curve 1) and its cooling after radiation termination (curve 2), and at that in this and subsequent figures the temperature changes $\Delta T$ were measured relative to the liquid temperature in the initial state before the experiment began. 


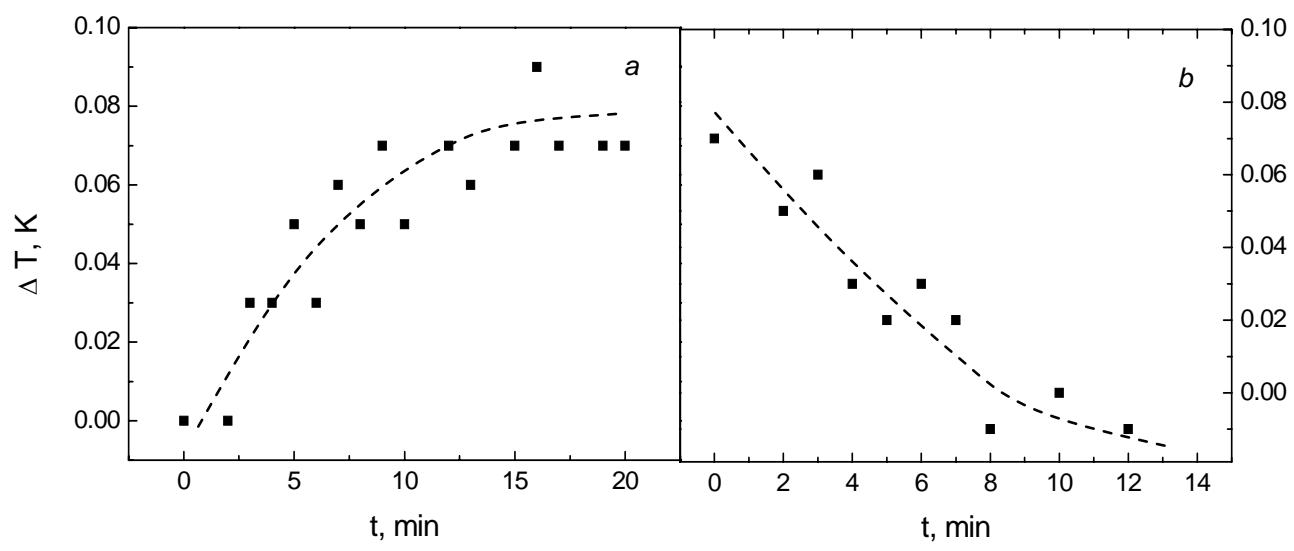

Fig. 1. Time dependences of water heating under the action of cyclotron radiation from Ge ingot $(a)$ and its cooling after radiation termination $(b)$.

Fig. 2 shows the dependence of the change in temperature on time upon cooling water with the volume $30 \mu \mathrm{l}$ under the action of cyclotron radiation from the germanium ingot (curve 1). After termination of cyclotron radiation (removal of the ingot from the magnet gap), the obtained state was preserved for an hour or more (curve 2). With the repeated action of cyclotron radiation, the water temperature tended to the initial value corresponding to the start of the experiment (curve 3).

In studies, the time dependences were recorded as well, in which two competing effects manifested themselves: first heating, and then cooling the liquid under the action of radiation. Fig. 3 shows the corresponding dynamics of temperature changes of water with the volume $30 \mathrm{ml}$ under the action (curve 1), after cessation of action (curve 2) and with the repeated action (curve 3) of cyclotron radiation from a germanium ingot.

Let us discuss the results obtained.

When a semiconductor crystal is placed in a homogeneous magnetic field with a constant induction $B$, taking into account that the effective mass of holes is a scalar quantity (isotropic mass), hole begins to move along spiral trajectories, i.e., helical lines, axis of which coincides with the induction magnetic field. This means that the particle simultaneously participates in two motions: under the action of the Lorentz force it rotates uniformly at the velocity $v_{\perp}$ along the circle $\left(v_{\perp}\right.$ is the component of the hole thermal velocity perpendicular to the vector of magnetic induction) and it is translation movement under its own inertia, i.e. moves uniformly and rectilinearly at a constant velocity $v_{\|}$that is a component of the hole thermal velocity parallel to the vector of magnetic induction.

The angular velocity of hole rotation is called the cyclotron (Larmor) frequency $\omega_{B}$. For nonrelativistic particles (for holes in a semiconductor crystal, the thermal velocity is much lower than the speed of light), it is equal to [1]:

$\omega_{B}=\frac{e B}{m_{p}}$,
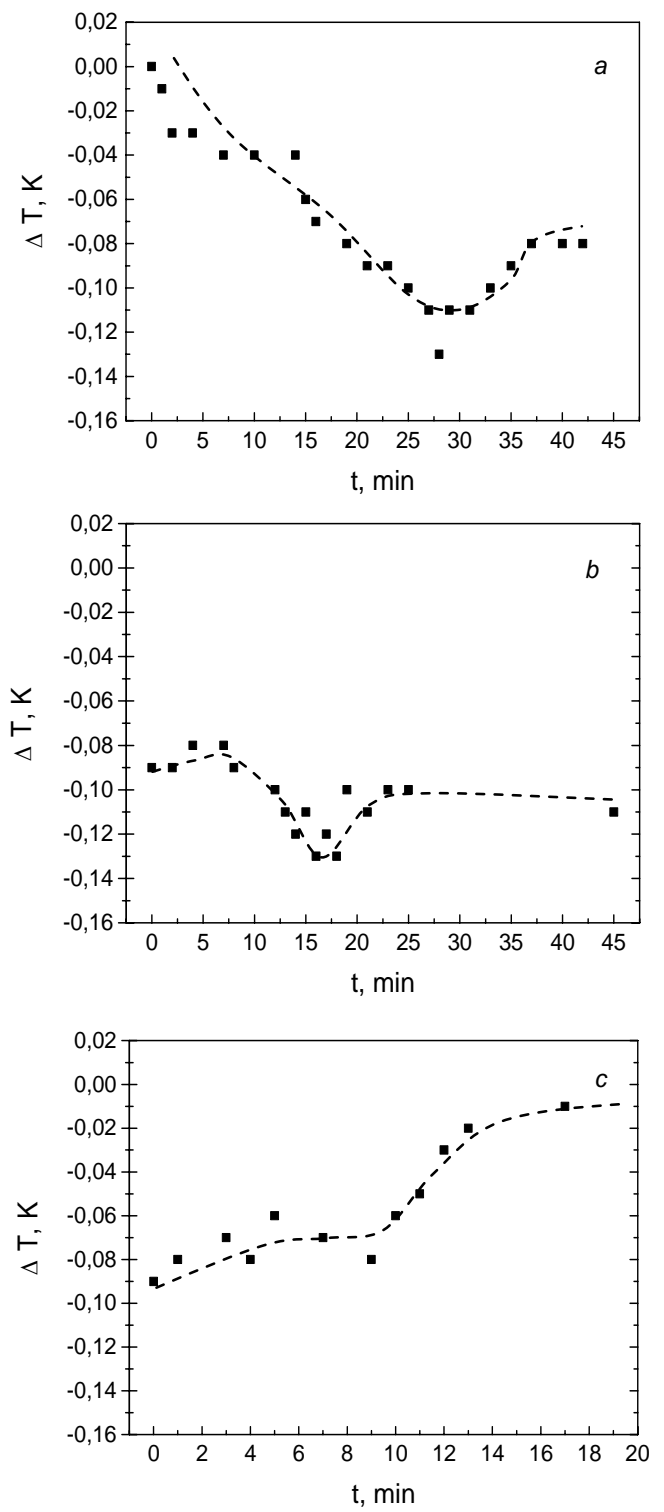

Fig. 2. Time dependences of changes in the water temperature: $a$ - under the action of cyclotron radiation from Ge ingot; $b-$ after radiation termination; $c$ - under the repeated action of radiation. 
where $e$ is the elementary electric charge (electron charge). At $B=0.45 \mathrm{~T}$, the cyclotron frequency of light holes $\left(m_{p}=0.043 m_{0}\right.$, where $m_{0}$ is the free electron mass), accordingly to the equation, equals $v_{B}=$ $2.9 \cdot 10^{11} \mathrm{~Hz}\left(\omega_{B}=2 \pi v_{B}\right)$.

Since during rotation the particle undergoes acceleration that is constant in magnitude and directed perpendicularly to the velocity, it is the source of radiation of electromagnetic waves at the frequency $\omega_{B}$ [1-3].
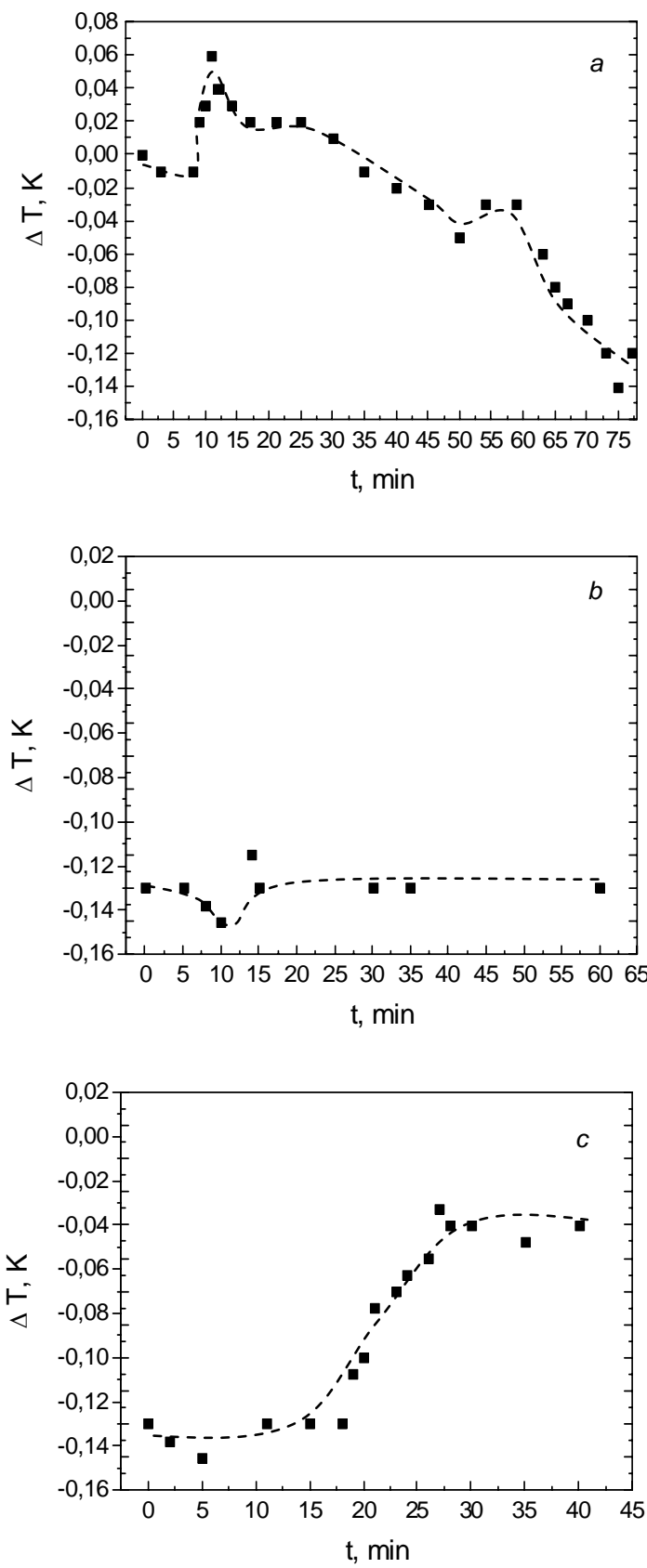

Fig. 3. Time dependences of changes in the water temperature: $a-$ under the action of cyclotron radiation from Ge ingot; $b-$ radiation is absent; $c$ - repeated radiation.
Radiation of all charged carriers still determines the intensity of the cyclotron radiation of thermal plasma in a semiconductor crystal. However, not all the semiconductor crystal radiates, but only some its near-surface region does it. Radiation in the bulk is "locked".

In the bulk of a semiconductor crystal, where this radiation is "locked", it is in a thermodynamically equilibrium state with matter. That is, radiation of charged particles is compensated by absorption. In this case, the radiation spectrum of plasma is equivalent to that of the absolutely black body $[1,2]$. In the outer layers, from the fact that the radiation yield becomes significant, plasma is in a state of local thermodynamic equilibrium, and radiation is not the same as the blackbody one. Its spectrum is localized in the vicinity of the cyclotron frequency, and the radiation intensity does not exceed that for the absolutely black body in vacuum [1].

Let's suppose that plasma of the outer radiating layer is not in a state of local thermodynamic equilibrium. In particular, the fact of presence of nearsurface space charge regions, which are depleted of free electrons or holes, is essential for this situation. These regions, due to the above circumstance, also have a reduced ability to absorb electromagnetic waves. In view of this, the space charge region is an effective drain of photons from the radiating layer of the semiconductor into the surrounding space. As a result, the cyclotron radiation of the crystal outer layer is not compensated by absorption, that is, the time for achieving an equilibrium particle energy distribution exceeds the time of energy losses by radiation. In other words, the cyclotron radiation of thermal electrons becomes thermodynamically non-equilibrium. As a consequence, the near-surface region of semiconductor is cooled. In [4], the surface of Ge, InSb, InAs, and GaAs was cooled after exposure to magnetic field pulses.

The degree of deviation of plasma from the state of thermodynamic equilibrium has a limit. The energy of a cyclotron radiation quantum should not exceed the average kinetic energy of the thermal motion of charge carriers.

The effect of cooling the liquid under the action of cyclotron radiation and the subsequent preservation of the achieved state after cessation of the action of electromagnetic radiation can be interpreted as the effect of the "memory" of water. That is, the liquid "remembers" the fact of its irradiation $[5,6]$ and can serve as a carrier of information, acquiring new properties under the influence of low-intensity millimeter radiation, that are responsible to a therapeutic effect observed when using previously irradiated water $[6,7]$. It is based on the resonance interaction of radiation with proton and cluster structures $\left(\mathrm{H}_{2} \mathrm{O}\right)_{n}$ (the values lie within the range from 2 up to 140), the spectrum of own frequencies of which comprises in the millimeter and submillimeter regions [6, 8, 9]. Electromagnetic radiation causes destruction of cluster conglomerates due to resonant phenomena as well as subsequent restoration in another form, which leads to a 
change in the hydrogen bonds between the molecules of water and, hence, the molecule itself [8]. In particular, a shift of signal in the proton nuclear magnetic resonance to a strong field is observed, which indicates destruction of hydrogen bonds between water molecules followed by the passage of molecules from liquid to vapor [8]. In water, for any frequency of electromagnetic waves in the superhigh-frequency and terahertz ranges, there are always clusters capable to absorb radiation quanta [6]. Metastable states formed in water under action of radiation persisted from 2-3 days to several weeks [6, 8].

Thus, destruction of cluster conglomerates intensifies the process of water evaporation. As a result, the temperature of the near-surface layer of liquid is lowered, where the thermocouple is located. Repeated action of cyclotron radiation makes it possible to reverse formation of cluster structures, which is accompanied by a decrease in the intensity of evaporation and an increase in the temperature of liquid to its initial state.

Finally, the presence of a section corresponding to water heating is likely to be strongly influenced by the fact that the magnetic induction in the air gap is distributed depending on the radial coordinate, and in the case of the ingot accidental location, some deviations in the values of the cyclotron frequency take place in it. Apparently, when the cyclotron frequency coincided with one of the eigenfrequencies of the resonator, the heating effect took place.

\section{Conclusion}

Magnetized thermal plasma of the radiating layer under certain circumstances, in particular caused by the presence of a space-charge region at the surface of semiconductor crystal, is not in the state of thermodynamic equilibrium. In this case, under the action of magnetic field, transformation of kinetic energy of free carrier thermal motion into cyclotron radiation of microwave and terahertz ranges, which is not in thermodynamic equilibrium with material, takes place. Energy of electromagnetic radiation quantum is limited by the average kinetic energy of free carriers thermal motion. The latter circumstance limits the degree of deviation of plasma from the state of thermodynamic equilibrium. Smooth tuning the radiation frequency can be carried out by changing the magnetic field induction.

\section{References}

1. Bekefi G. Radiation Processes in Plasma. New York: Wiley, 1966.

2. Krall N.A., Trivelpiece A.W. Principles of Plasma Physics. New York, McGraw-Hill Book Company, 1973.

3. Longmire C.L. Elementary Plasma Physics. New York and so on: Intersci. Publ., 1963.

4. Davydov V.N., Loskutova E.A., Naiden E.P. Delayed structural changes in semiconductors induced by magnetic field. Fizika i tekhnika poluprovodnikov. 1989. 23, Issue 9. P. 1596-1600 (in Russian).

5. Fesenko E.E., Geletyuk V.I., Kasachenko V.N., Chemeris N.K. Preliminary microwave irradiation of water solution changes their channel-modifying activity. FEBS Lett. 1995. 366. P. 49-52.

6. Betskiy O.V. Pioneer works on millimeter electromagnetic biology, performed in IRE RAS. Biomedical radioelectronics. 2003. No. 8 . P. 11-20.

7. Deviatkov N.D., Kislov V.Ya., Kolesov V.V., Smirnov V.F., Chigin E.P. Revealing the effect of normalization in functional state of man's viscera under the action of water activated by millimeter radiation. Millimeter waves in biology and medicine. 1996. No. 8. P. 65-68.

8. Gapochka L.D., Gapochka M.G., Korolev A.F., Kostenko A.I., Suhorukov A.P., Timoshkin I.V. Effect of electromagnetic irradiation of extremely high and superhigh frequencies on liquid water. Bulletin of Moscow University. Ser. 3. Physics, Astronomy. 1994. 35, No. 4. P. 71-76.

9. Buffay I.P., Brown W.B., Gebbie H.A. Icosahedral water clusters. Chem. Phys. Lett. 1988. 148, No. 4. P. 281-284. 